\title{
A Meta-Analysis of the Conditions and Materials of Bone Grafting in High Tibal Osteototomy
}

\author{
Wei He ${ }^{1}$, Haojie Chen ${ }^{2}$, Min Wang ${ }^{2}$, Xiaoqiang Huang, ${ }^{2,3, *}$ \\ ${ }^{1}$ Shaanxi University of Trditional Chinese Medicine, Xianyang, 712046, China. \\ ${ }^{2}$ Department of Orthopedics, Xi'an Honghui Hospital, Xi'an, 710001, China. \\ ${ }^{3}$ Department of Orthopedics, Xi'an Fifth Hospital, Xi'an, 710001, China.
}

How to cite this paper: Wei He, Haojie Chen, Min Wang, Xiaoqiang Huang. (2021) A Meta-Analysis of the Conditions and Materials of Bone Grafting in High Tibal Osteototomy. International Journal of Clinical and Experimental Medicine Research, 5(1), 75-82.

DOI: $10.26855 /$ ijcemr.2021.01.010

Received: December 28, 2020

Accepted: January 22, 2021

Published: January 29, 2021

*Corresponding author: Xiaoqiang Huang, Department of Orthopedics, Xi'an Honghui Hospital, Xi'an,

710001, China; Department of Orthopedics, Xi'an Fifth Hospital, Xi'an, 710001, China.

Email: huangxq73@163.com

\begin{abstract}
Nonunion or delayed union of osteotomy gay in open wedge high tibial osteotomy is a common postoperative complication. Bone grafting is generally not required in the osteotomy gay. Bone grafting should be considered in patients aged more than 46 years oid, with lateral hinge fractures, with a height of more than $14 \mathrm{~mm}$ in the osteotomy gay distraction, or with a corrected varus deformity exceeding $12^{\circ}$. Autogenous iliac graft is still considered the "gold standard" in the osteotomy gay. In conclusion, open wedge high tibial osteotomy using different types of bone grafting materials and rigid internal fixation is one of the successful surgical methods for the treatment of medial compartment osteoarthritis with varus deformity.
\end{abstract}

\section{Keywords}

Opening wedge, High tibial osteotomy, osteotomy gap, Bonegraft

Knee osteoarthritis (KOA) which is mainly characterized by varus deformity and articular cartilage wear, causes pain and dysfunction, and requires joint replacement surgery at an advanced stage. High Tibial osteotomy (HTO) is one of the most important surgical means of treating KOA. At present, there are mainly two ways: Inside open wedge high tibial osteotomy (OWHTO) and the lateral closing wedge osteotomy (CWHTO), due to the need of CWHTO to additional bone cutting fibula, and there is lead to the potential risk of peroneal nerve injury and other complications, in recent years, OWHTO become more and more popular, especially in the medial compartment before mainly mild genu varus deformity of KOA. It shows a 53\%-93\% 10-year survival rate [1]. OWHTO provides accurate correction through progressive openings, standardized exposure and the avoidance of lateral knee joints such as tibial peroneal proximal joint fractures and common peroneal nerve palsy, compartment syndrome, as well as early post-operative full weight bearing. Disadvantages include increased tibial slope, risk of reduced patella height, possible need for bone grafting in the gaps formed at the osteotomy, and problems resulting in delayed or uncorrectable bone healing $[1,2]$.

\section{Influencing factors of osteotomy nonunion and delayed healing Current factors}

Influencing factors of osteotomy nonunion and delayed healing Current factors that resulted in delayed or nonunion of osteotomy gap after OWHTO included smoking, obesity (Mean body-mass index, Mean body-mass index), age, weight-bearing time, a lateral hinge fracture (LHF), size of disconnection gap distraction, type of fixation device and other factors. Some factors are listed as follows: 


\subsection{Smoking}

Braun et al. [3] found the mechanism of action by which smoking delayed bone healing: smoking damaged bone cells and their homeostasis by increasing the formation of reactive oxygen species. The survival rate of human osteoblasts decreased in concentration and time dependence. Therefore, cigarette smoke causes damage to human osteoblasts through the accumulation of reactive oxygen species. However, Floerkemeier et al. [4] showed no association between smoking and delayed bone healing.

\subsection{Obesity}

Increased BMI was significantly associated with delayed consolidation and lateral tibial hinge fractures. In the relevant literature, the results were inconsistent, and Meidinger [5] found a correlation between the two. Previous studies have found that increased BMI is associated with greater correction loss and greater correction Angle [6]. In contrast, the current study found that these two factors had no effect on the healing of the osteotomy gap [7].

\subsection{Lateral hinge fracture}

Biomechanical studies have shown that OWHTO intraoperative or postoperative LHF can reduce the stability of bone structure because it leads to excessive micromotion and is a major risk factor for delayed healing of bone space and failure of correction, especially when unstable plates are used for fixation [8]. Miller et al. [9] pointed out in their report that if LHF is not detected or treated, its complications are likely to lead to correction loss and delayed or nonunion of osteotomy.

\subsection{Age}

Regarding age, Flecher et al. [10] reported \& GT; Age 50 was a risk factor for failure $(P=0.014$; Or, 2. 1). Khoshbin et al. [11] believed that OWHTO survival was related to age, and the failure rate of patients over 46 years old increased by 5\% per year [hazard ratio $=1.05(95 \%$ CI 1. 04, 1. 06), P < 0. 001)].

\subsection{The load time}

Studies have shown that early use of locking plates after full weight loading improves prognosis, similar to delayed weight loading in terms of clinical and radiological outcomes. In contrast, early loading was more advantageous in some radiological parameters and complications than delayed loading. However, the efficacy and safety of early postoperative load bearing still need further study [12]. Early full weight bearing starting 2 weeks or within 2 weeks after OWHTO fixation has been shown to be safe, does not lead to correction loss, and has a bone healing time similar to that of patients recovering with a standard 6-week rehabilitation regimen [13]. Takeuchi et al. [8] have determined that early weight-bearing exercise programs can promote eventual full weight-bearing 2 weeks after surgery. Of the 93 patients in their cohort, 87 were fully weight-bearing 2 weeks after surgery, while the remaining 6 were not fully weight-bearing due to various complications [2 exceptions for lateral plateform fractures, 2 cases of LHF(type III), and 2 cases of infection]. However, no implant failure, artificial wedge collapse, or screw loosening, with or without LHF was observed. They believe that an important factor in this situation is the use of the TomoFix plate in combination with the artificial bone replacement plate, because in this case the stress applied to the osteotomy site is distributed throughout the artificial bone replacement wedge and the TomoFix plate. In the case of tibial LHF, they recommend that full load not be allowed until callus has formed at the osteotomy site. Studies have shown that early use of locking plates after full weight

\subsection{Fixed type and early load bearin}

The fixation materials commonly used by OWHTO include anatomical T-plate, short-segment locking plate, and long-segment locking plate, etc., provide stable mechanical support for the osteotomy gap and promote bone healing. T-shaped plates with internal anatomy of the upper tibia are classified into non-locking and locking, and are often used in internal fixation of proximal tibial fractures. Compared with non-locking plate, locking plate can better maintain the stability of osteotomy end to promote osteotomy gap healing and avoid orthopedic Angle loss due to its angular stability. Therefore, the use of locking plate in OWHTO has gradually become a mainstream option. The long segment locking plate is represented by the Tomofix plate [14], with a T-shaped appearance, and there are 4 screws at the proximal end. The osteotomy area is the non-porous area of the plate. A Puddu plate is represented by a short-segment locking plate with a raised insertion space in the middle and a 4-hole locking screw inserted above and below. Compared with short segment locking plate, patients using long segment locking plate were con- 
scious of subcutaneous foreign body sensation and even irritation sensation. Therefore, in recent years, more and more research reports have been published on short segment locking plate [15]. Pornrattanamaneewong et al. [16] reported that when OWHTO interosteotomy was fixed with non-locking plate, tricortical ilium graft could promote the bone healing of interosteotomy, while Tomofix plate could achieve satisfactory curative effect without bone graft. In a biomechanical study, Stoffel [17] compared Puddu and TomoFix plates and found that the TomoFix plates showed significantly higher stability in torsion and compression when used in LHF. The mechanical stability of the long locking plate is proved.

\section{Treatment and correction of osteotomy space}

The more severe the varus deformity in KOA patients, the greater the OWHTO deformity correction Angle (i.e., the greater the gap distance between osteotomy and distraction), and the higher the risk of LHF. LHF may lead to instability of the lateral interosteotomy space and delayed or nonunion of interosteotomy bone. Previous studies reported: When the height of medial distraction is over $10 \mathrm{~mm}$, or the correction of varus deformity is over $12^{\circ}$, or LHF, or the patient has a high risk factor of non-union fracture, such as obesity, smoking, etc., bone grafting should be performed at the same time of firm internal fixation. Bone grafting is not recommended for OWHTO of less than $10 \mathrm{~mm}$. However, El-Assal et al. [18] reported that the medial clearance could be up to $14 \mathrm{~mm}$ without the use of bone cavity fillings, and advocated that osseous clearance fillings should be used only when the clearance was greater than $14 \mathrm{~mm}$.

In addition, bone healing was found to be dependent on gap size. If the space is smaller, the bone healing rate will be faster.

\section{Bone space filler selection}

At present, delayed or nonunion and correction of loss of OWHTO osteotomy space remains an important clinical problem. Therefore, there are a variety of materials and methods for filling bone space. A good bone graft material should have the following characteristics: (1) provide a scaffold for bone penetration and replacement by crawling; (2) Can promote osteoblasts to conduct new bone formation; (3) It can promote bone-induced biological proteins to promote differentiation and proliferation of osteoblasts; (4) It can provide structural support and early stress sharing at the site of wedge-shaped osteotomy defect, which is beneficial to bone healing and early functional exercise. In terms of biological activity and structure, the bone graft materials can be classified as: autoilium, autoosteophyte, fresh frozen or freeze-dried allograft, and bone replacement materials.

\subsection{Autologous bone}

In three cortical iliac bone autograft has enhanced bone induction and bone conduction, osteogenesis function, no immune response and economic advantages, is recognized as the best bone graft material, autogenous bone in bone induction and bone crawling is superior to other bone graft material, porous structure, can be compatible with the surrounding tissue rapid integration, is advantageous to the nutrient transfer and micro vascular reconstruction, in the early bone graft can be mixed with host bone defects and provide good mechanical support. Lash et al. [19] systematically evaluated and analyzed OWHTO interosteotomy bone grafts, and found that the rate of delayed bone union/non-union in the interosteotomy space after autologous iliac graft, allograft and osteogenic substitute graft was $2.6 \%, 4.6 \%$ and $4.5 \%$, respectively. This study suggested that the rate of delayed union/nonunion was the lowest in the osteotomy space when autoiliac graft was used compared with allograft and osteogenic substitute.

However, the incidence of complications at the donor site of autologous bone graft is $10 \%$ [20], and the risks of long-term pain, bleeding, increased operation time, numbness on the outside of the thigh, and infection are all serious challenges faced by autologous bone graft. Lim et al. [21] reported that proximal tibia from cortical cancellous bone is a feasible option, and that the amount of bone taken is the same or more than that of ilium bone, with low incidence of complications and less surgical trauma. Even with a large osteotomy with a lateral approach diameter of $25 \mathrm{~mm}$, the tibia can withstand three times the weight of the body. However, due to the limited exposure and the impact of vigorous exercise on the osteotomy site, the clinical surgeon can determine the size of the cortical window based on the patient's bone quality.

In conclusion, although there are complications associated with bone removal, autogenous iliac graft has all the basic characteristics of rapid and effective bone healing, such as strong bone induction and bone conduction, and osteogenesis, and should still be regarded as the gold standard in cases of non-union or delayed union with high risk. Proximal tibia can make up for the deficiency of autogenous iliac bone, which is a feasible alternative to autogenous iliac bone. 
In three cortical iliac bone autograft has enhanced bone induction and bone conduction, osteogenesis function, no immune response and economic advantages, is recognized as the best bone graft material, autogenous bone in bone induction and bone crawling is superior to other bone graft material, porous structure, can be compatible with the surrounding tissue rapid integration, is advantageous to the nutrient transfer and micro vascular reconstruction, in the early bone graft can be mixed with host bone defects and provide good mechanical support. Lash et al. [19] systematically evaluated and analyzed OWHTO interosteotomy bone grafts, and found that the rate of delayed bone union/non-union in the interosteotomy space after autologous iliac graft, allograft and osteogenic substitute graft was $2.6 \%, 4.6 \%$ and $4.5 \%$, respectively. This study suggested that the rate of delayed union/nonunion was the lowest in the osteotomy space when autoiliac graft was used compared with allograft and osteogenic substitute. However, the incidence of complications at the donor site of autologous bone graft is 10\% [20], and the risks of long-term pain, bleeding, increased operation time, numbness on the outside of the thigh, and infection are all serious challenges faced by autologous bone graft. Lim et al. [21] reported that proximal tibia from cortical cancellous bone is a feasible option, and that the amount of bone taken is the same or more than that of ilium bone, with low incidence of complications and less surgical trauma. Even with a large osteotomy with a lateral approach diameter of $25 \mathrm{~mm}$, the tibia can withstand three times the weight of the body.

However, due to the limited exposure and the impact of vigorous exercise on the osteotomy site, the clinical surgeon can determine the size of the cortical window based on the patient's bone quality.

In conclusion, although there are complications associated with bone removal, autogenous iliac graft has all the basic characteristics of rapid and effective bone healing, such as strong bone induction and bone conduction, and osteogenesis, and should still be regarded as the gold standard in cases of non-union or delayed union with high risk. Proximal tibia can make up for the deficiency of autogenous iliac bone, which is a feasible alternative to autogenous iliac bone.

\subsection{Autologous osteophyte}

Osteophytes are physiological osteophytes that grow at the edge of the joint surface during the development of osteoarthritis. It is associated with active endochondral bone formation and the expression of various growth factors. Dodds and Gowen [23] report that osteophytes contain all components of the bone remodeling cycle, including the endochondral ossification process. Some researchers have proposed that bone morphogrowth protein plays an important role in the growth and development of bone and has the ability to induce and promote the formation of cartilage and bone in the ectopic sites [22]. Due to its potential strong bone induction effect, it can be the source of bone transplantation.

Active and passive quadriceps strengthening exercises can be initiated 2 days after autosectomous bone removal. Patients were allowed to use 2 crutches to begin partial weight bearing 1 week after surgery. Full weight bearing is allowed 4 weeks after surgery. Three months after the operation, the patients were allowed to resume normal activities, including jogging [24]. El-assal et al. [18] reported that autologous osteophyte was used to confirm bone healing by radiography on average 3 months after surgery (12. 4 weeks). At the same time, Brosset et al. [25] conducted X-ray examination of 51 OWHTO patients without bone grafting, and observed the bone healing status in an average of 4.5 months after surgery. These two studies suggest that autologous osteophyte promotes bone healing in the osteotomy site of the knee joint better than that of the knee joint without bone grafting. Meanwhile, osteophyte grafts are readily available under arthroscopy compared with autogenous ilium grafts, resulting in shorter bone healing time, no additional skin incisions required, and improved range of motion. However, bone fragments and long-term blood accumulation in the knee joint may be drawbacks of this technique [24].

\subsection{Allograft bone}

Compared with autologous bone, allograft bone can be used to fill the osteotomy space with abundant sources, no limitation in shape or size, and its bone conductivity, biological characteristics and morphological structure are the closest to autologous bone. It can be stored and transported for a long time, shorten the operation time during operation, and avoid the surgical risk of ilium extraction from the donor area.

Allograft bone is the most commonly used material to replace autograft bone due to its integrity structure, mechanical stability, biological activity, bone induction and low immunity, as well as strong healing ability with host bone. Allogeneic bone is an allogeneic biological material with certain immune antigenicity, accompanied by potential risks of immune response or disease transmission. At present, reducing its immune rejection is the key to improve the effect of transplantation therapy.

Santic et al. [27] found that the healing time of larger allografts was significantly longer than that of smaller al- 
lografts. At 12 weeks, the healing rates of allograft cancellous bone were $90 \%, 47 \%$, 6\% when the osteotomy gap was $\leq 9 \mathrm{~mm}, 10 \mathrm{~mm}$, and 11-16 mm. The locking plate with angular stability should be selected for the internal fixation device when allogeneic bone graft is applied to the WHTO osteotomy gap to avoid osteotomy Angle loss.

\subsection{Bone replacement material}

Various synthetic bone substitutes have been introduced to replace autologous bone grafts to fill the open space. Their advantages are that fillers are easy to obtain and use and have bone conduction function, which can theoretically replace autologous bone and avoid the disease of the donor site. The degradation rate and absorptivity of different bone replacement materials are different, and the effect and time of promoting bone healing are also different. The ideal artificial bone should be compatible with tissue, non-toxic, have good osteogenic effect, have certain mechanical strength, and be able to degrade in time and finally be completely replaced by autogenous bone tissue.

Bone substitution materials currently used include demineralization of bone matrix, hydroxyapatite (HA), Coralling Hy Drox y Aptite (CHA), tricalcium Phosphoates,TCP), calcium-phosphate Cement (CPC), bone morphogenetic proteins (such as BMP-2 and BMP-7), Biphasic Calcium phosphates (BCP), Calcium phosphate cement, Calcium sulfate, ceramics, bioactive glass, etc.

The chemical composition of HA is basically similar to that of human bone and has good biocompatibility. However, HA was once considered as a biodegradable material. Wang Hanxu et al. [21] reported that 31 KOA patients were treated with OWHTO, and single porous HA was fixed with long locking TomoFix plate after osteotomy space transplantation. The study found that HA could play a structural support role in the early postoperative period to enhance the stability of internal fixation, but its disadvantage was that its autogenous absorption process was slow. CHA [27] is a kind of hydroxyapatite material which is processed with specific Marine corals as raw material. Compared with HA, it not only has excellent biocompatibility and bone affinity, but also has a three-dimensional structure similar to that of human bone, which can provide a suitable space for the growth of new bone. But, like HA, it has the disadvantage of being absorbed very slowly by the body. The absorbable coral hydroxyapatite (R-CHA), made of hydroxyapatite and calcium carbonate, compensates for the slow absorption of HA and CHA in the body. After R-CHA is implanted in the body, it can mediate good fracture healing, and can be gradually degraded and absorbed within the expected time. Therefore, R-CHA is expected to be an ideal bone graft replacement material and bone tissue engineering scaffold material [27]. Animal experiments and clinical applications have proved that this composite artificial bone has good histocompatibility and no systemic or local toxicity, and can be safely applied to repair various bone defects with superior osteogenic ability, making it a good bone graft substitute. Long-term X-ray observation and follow-up of the composite artificial bone graft showed that the mass gallery with high density was still not completely replaced after 24 months. Therefore, the understanding and control of the degradation rate of the artificial bone remains to be further studied.

CPC is a non-ceramic form of hydroxyapatite artificial bone material. After CPC is implanted, an induction membrane is formed in the local tissue of the outer bone membrane, providing a closed space for the implanted autogenous bone particles to bond with the host. However, the intramedullary mass effect of CPC would hinder fracture healing [28]. Therefore, the Human bone Morphogeneticprotein 2-loaded calcium phosphate cement (CPC/RHBMP-2) synthesized by CPC and RHBMP-2 is regarded as a new type of bone tissue repair and reconstruction material. The results showed that CPC/RHBMP-2 had stronger vascularization and bone-promoting effects than CPC or RHBMP-2 alone [29].

TCP artificial bone is a theoretically ideal bone graft material, which has the advantages of biocompatibility, mechanical tolerance and biodegradability. Lee DY et al. [30] randomly assigned 54 patients who had received OWHTO treatment for varus genu and OA to two groups. TCP particles were used in 27 cases, and freeze-dried allogeneic bone slices were used in 27 cases. The degree of bone healing was scored on a five-point scale at 6 weeks, 3 months, 6 months, and 12 months after surgery with knee planks.

Six months and 12 months after surgery, the highest degree of bone healing was grade 4 . There was no statistical difference in the number of progressive cases of bone healing between the two groups at each time point $(\mathrm{P}>$ 0.05). There were no significant differences between the two groups in preoperative and postoperative MPTA, correction Angle, and wedge size. No complications such as corrected loss, nonunion, hardware failure, wound infection, or deep vein thrombosis were observed in both groups during the one-year follow-up period. In OWHTO, there was no radiological or clinical difference in the rate of progressive bone healing using TCP particles and allograft bone chips as bone filling substitutes. TCP particles can induce immune response, rarely increase the risk of disease transmission, and overcome some disadvantages of allograft chip transplantation. TCP particles are a good substitute for allograft chip. 
PRP, a factor that enhances bone healing, is readily available from the patient's blood and contains higher concentrations of platelets and fibrinogen, as well as growth factors. PRP had therefore been expected to enhance wound healing and reduce bone exposure by showing platelets as procoagulant agents. However, there is no evidence in the current literature that the addition of PRP promotes bone healing.

Aryee et al. [31] reported that OWHTO was used to treat 60 KOA patients, which were divided into 3 groups on average: Group A was boneless graft group, group B was wedge-shaped HA/TCP bone substitute graft group, and group C was wedge-shaped HA/TCP bone substitute combined with PRP group. Postoperatively a Puddu locking plate is used. Osteotomy and orthopedic Angle FTA from inward $(5.3 \pm 0.8)^{\circ}$ to outward $(1.9 \pm 0.4)^{\circ}$; Callus growth was observed laterally and posteriorly 6-8 weeks after operation. Follow-up at 12 months after the operation showed that wedge HA/TCP contours were still visible in the osteotomy space on X-ray, and there was no difference in clinical functional results between groups B, C and A. When the steel plate was removed 12 months after the operation, $5 \mathrm{~mm}$ tissue biopsies were performed in the osteotomy gap, and the growth of new bone cells and HA/TCP residues were found in both Group B and C. Therefore, it is inferred that in osteotomy orthotics \& LT.

For patients at $10^{\circ}$, the osteotomy space was treated with bone replacement transplantation, which was not better than the boneless group. In patients with osteotomy and orthopedic $\geq 10^{\circ}$, bone replacement material transplantation combined with PRP was used in the osteotomy space, and its efficacy remains to be further studied. Even if PRP presents limited risk of infection and adverse reactions due to its source (autologous blood), it can only be used as a supplement to other materials and not as an independent bone substitute.

\section{Conclusion and Prospect}

To sum up, OWHTO is one of the important surgical methods for the treatment of the former medial compartment mainly KOA. Influenced by smoking, obesity, age, weight-bearing time, LHF, size of interosseous distraction, type of fixation device and other factors, delayed/non-union of interosseous union is a common complication.

OWHTO generally does not require bone grafting. Bone grafting should be considered when patients are 46 years old of >, LHF, $14 \mathrm{~mm}$ of osteotomy space $>$, or the corrected varus deformity is more than $12^{\circ}$. With the feature of angular stability, Tomofix, as the representative of the long segment locking plate, can better maintain the stability of the osteotomy end, promote the osteotomy gap healing, and avoid orthopedic failure. In the selection of bone graft materials, the autogenous ilium graft should still be regarded as the gold standard in the case of non-union or delayed union with high risk due to all the basic characteristics of rapid and effective bone healing, despite the complications associated with bone removal. Proximal tibia bone grafting can make up the defect of autograft bone grafting and is a feasible alternative to autograft ilium bone grafting. Compared with autologous bone grafting, autologous osteophyte has the advantages of convenient acquisition and no additional skin incision for interosteotomy space bone healing, which is better than the knee joint without bone grafting. Fragmental cancellous bone fixation combined with locking plate can achieve better results in allograft bone transplantation, and the smaller the osteotomy gap is, the faster the healing rate will be. R-CHA, TCP, AND BCP are the ideal materials for artificial bone substitutes to repair bone defects. Whether factors that enhance bone healing components, such as BMP and PRP, contribute to OWHTO osteotomy space healing remains to be confirmed by further clinical studies.

In conclusion, the osteotomy gap bone healing was closely related to the size of the distraction gap, age, LHF, type of fixation device, obesity, and weight-bearing time, etc. Different types of bone graft materials have their advantages and disadvantages, but no matter what type of filler is used, it can improve the function of KOA and relieve pain. A better OWHTO interosteotomy treatment requires a lot of clinical data to be determined and the surgeon to weigh it against the patient's physical condition.

\section{Acknowledgements}

This study was approved by Key research and development project of Shaanxi Province (2018SF-195).

\section{References}

[1] Dubrana, F., Lecerf, G., Nguyen-Khanh, J. P., et al. (2008). Tibial valgus osteotomy. Rev Chir Orthop, 94(4 Suppl): S2-21.

[2] Pipino, G., Indelli, P. F., Tigani, D., Maffei, G., Vaccarisi, D. (2016). Opening-wedge high tibialosteotomy: a seven-to twelve-year study. Joints, 4: 6-11.

[3] Braun, K. F., Ehnert, S., Freude, T., et al. (2011). Quercetin protects primary human osteoblasts exposed to cigarette smoke through activation of the antioxidative enzymes HO-1 and SOD-1. Scientific World Journal, 11: 2348-2357. 
[4] Floerkemeier, S., Staubli, A. E., Schroeter, S., Goldhahn, S., Lobenhoffer, P. (2014). Does obesity and nicotine abuse influence the outcome and complication rate after open-wedge high tibialosteotomy? A retrospective evaluation of five hundred and thirty three patients. Int Orthop, 38: 55-60.

[5] Meidinger, G., Imhoff, A. B., Paul, J., Kirchhoff, C., Sauerschnig, M., Hinterwimmer, S. (2011). May smokers and overweight patients be treated with a medial open-wedge HTO? Risk factors for nonunion. Knee Surg Sports Traumatol Arthrosc, 19: 333-339.

[6] Miller, B. S., Downie, B., McDonough, E. B., Wojtys, E. M. (2009). Complications after medial opening wedge high tibial osteotomy. Arthroscopy, 25(6): 639-646.

[7] Fucentese, S. F., Tscholl, P. M., Sutter, R., Brucker, P. U., Meyer, D. C., Koch, P. P. (2019). Bone autografting in medial open wedge high tibial osteotomy results in improved osseous gap healing on computed tomography, but no functional advantage: a prospective, randomised, controlled trial. Knee Surg Sports Traumatol Arthrosc., 27(9): 2951-2957.

[8] Takeuchi, R., Ishikawa, H., Kumagai, K., et al. (2012). Fractures around the lateral cortical hinge after a medial opening-wedge high tibial osteotomy: a new classification of lateral hinge fracture. Arthroscopy, 28(1): 85-94.

[9] van Houten, A. H., Heesterbeek, P. J., van Heerwaarden, R. J., van Tienen, T. G., Wymenga, A. B. (2014). Medial open wedge high tibial osteotomy: can delayed or nonunion be predicted? Clin Orthop Relat Res., 472: 1217-23.

[10] Flecher, X., Parratte, S., Aubaniac, J.-M., Argenson, J. N. (2006). A 12-28-Year Followup Study of Closing Wedge High Tibial Osteotomy. Clin Orthop Relat Res., 452: 91-6.

[11] Khoshbin, A., Sheth, U., Ogilvie-Harris, D., Mahomed, N., Jenkinson, R., Gandhi, R., Wasserstein, D. (2015). The effect of patient, provider and surgical factors on survivorship of high tibial osteotomy to total knee arthroplasty: a population-based study. Knee Surg Sports Traumatol Arthrosc.

[12] Lee, O. S., Ahn, S., Lee, Y. S. (2017). Effect and safety of early weight-bearing on the outcome after open-wedge high tibial osteotomy: a systematic review and meta-analysis. Arch Orthop Trauma Surg., 137(7): 903-911.

[13] Brinkman, J. M., Luites, J. W., Wymenga, A. B., van Heerwaarden, R. J. (2010). Early full weight bearing is safe in openwedge high tibial osteotomy. Acta Orthop, 81: 193-198.

[14] Aryee, S., Imhoff, A. B., Rose, T., Tischer, T. (2008). Do we need synthetic osteotomy augmentation materials for openingwedge high tibial osteotomy. Biomaterials, 29: 3497-3502.

[15] Passarelli, M. C., Filho, J. R. T, Brizzi, F. A. M., et al. (2017). Comparison of Puddu osteotomy with or without autologous bone grafting: a prospectiveclinical trial. Rev Bras Ortop, 52(5): 555-560.

[16] Pornrattanamaneewong, C., Harnroongroj, T., Chareancholvanich K. (2012). Loss of correction after medial opening wedge high tibial osteotomy: a comparison of locking plates without bone grafts and non-locking compression plates with bone grafts. J Med Assoc Thai, 95(Suppl 9): S21-S28.

[17] Stoffel, K., Stachowiak, G., Kuster, M. (2004). Open wedge high tibial osteotomy: Biomechanical investigation of the modified Arthrex Osteotomy Plate (Puddu Plate) and the TomoFix Plate. Clin Biomech (Bristol, Avon), 19: 944-950.

[18] El-Assal, M. A., Khalifa, Y. E., Abdel-Hamid, M. M., Said, H. G., Bakr, H. M. (2010). Opening-wedge high tibial osteotomy without bone graft. Knee Surg Sports Traumatol Arthrosc, 18: 961-966.

[19] Lash, N. J., Feller, J. A., Batty, L. M., Wasiak, J., Richmond, A. K. (2015). Bone grafts and bone substitutes for opening-wedge osteotomies of the knee: a systematic review. Arthroscopy, 31: 720-30.

[20] Alolabi, B., Dianne, B., Fowler, P. J., Willits, K., Giffin, J. R. (2011). Graft choice in medial opening wedge high tibial osteotomy: autovsallograft. Orthopaedicproceedings, 93-B(SUPP_IV): 581.

[21] Mauffrey, C., Madsenp, M., Bowles, R. J., Seligson, D. (2012). Bone graft harvest site options in orthopaedic trauma: a prospective in vivo quantification study. Injury, 43(3): 323-6.

[22] Bosemark, P., Isaksson, H., McDonald, M. M., Little, D. G., Tägil, M. (2013). Augmentation of autologous bone graft by a combination of bone morphogenic protein and bisphosphonate increased both callus volume and strength. Acta Orthop., 84(1): 106-111.

[23] Dodds, R. A., Gowen, M. (1994). The growing osteophyte: A model system for the study of human bone development and remodeling in situ. J Histotechnol, 17: 37-45.

[24] Akiyama, T., Okazaki, K., Mawatari, T, Ikemura, S., Nakamura, S. (2016). Autologous Osteophyte Grafting for Open-Wedge High Tibial Osteotomy. Arthrosc Tech., 5(5): e989-e995. Published 2016 Sep 5.

[25] Brosset, T., Pasquier, G., Migaud, H., Gougeon, F. (2011). Opening wedge high tibial osteotomy performed without filling the defect but with locking plate fixation (TomoFix) and early weight-bearing: Prospective evaluation of bone union, precision and maintenance of correction in 51 cases. Orthop Traumatol Surg Res., 97: 705-711.

[26] Santic, V., Tudor, A., Sestan, B., Legovic, D., Sirola, L., Rakovac, I. (2010). Bone allograft provides bone healing in the medial opening high tibial osteotomy. Int Orthop, 34: 225-229.

[27] Parizi, A. M., Oryan, A., Shafiei-Sarvestani, Z., et al. (2013). Effectiveness of synthetic hydroxyapatite versus Persian Gulf coral in an animal model of long bone defect reconstruction. J Orthop Traumatol., 2013 Dec, 14(4): 259-68. 
[28] Goldberg, V. M., Stevenson, S. (1987). Natural history of autografts and allografts [J]. Clin Orthop Relat Res., 225: 7-16.

[29] Lin, D., Zhang, J., Bai, F., et al. (2016). Fabrication and clinical application of easyto-operate pre- cured CPC/rhBMP-2 micro-scaffolds for bone regeneration [J]. Am J Transl Res., 8(3): 1379-1396.

[30] Lee, D. Y., Lee, M. C., Ha, C. W., et al. (2019). Comparable bone union progression after opening wedge high tibial osteotomy using allogenous bone chip or tri-calcium phosphate granule: a prospective randomized controlled trial. Knee Surg Sports Traumatol Arthrosc., 27(9): 2945-2950.

[31] Aryee, S., Imhoff, A. B., Rose, T., et al. (2008). Do we need synthetic osteotomy augmentation materials for opening-wedge high tibial osteotomy. Biomaterials, 29(26): 3497-3502. 